
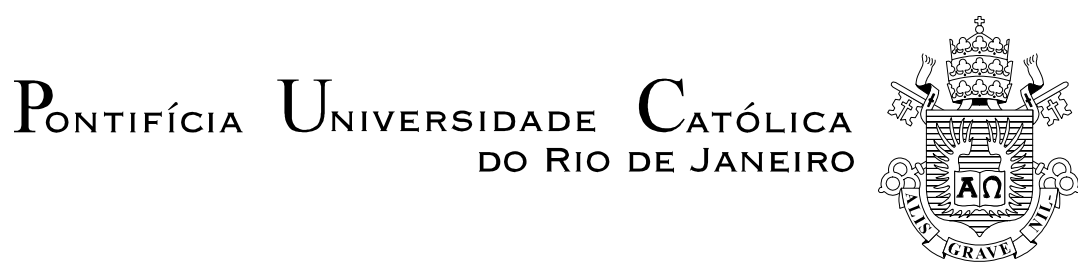

Fabíola Alice Alves Rocha dos Reis

Crenças sobre ensino e aprendizagem

de tradução: uma reflexão baseada nos Estudos da Tradução e na Linguística Aplicada

Dissertação apresentada como requisito parcial para obtenção do grau de Mestre pelo programa de Pós-Graduação em Letras do Departamento de Letras do Centro de Teologia e Ciências Humanas da PUC-Rio.

Orientadora: Prof. Marcia do Amaral Peixoto Martins 


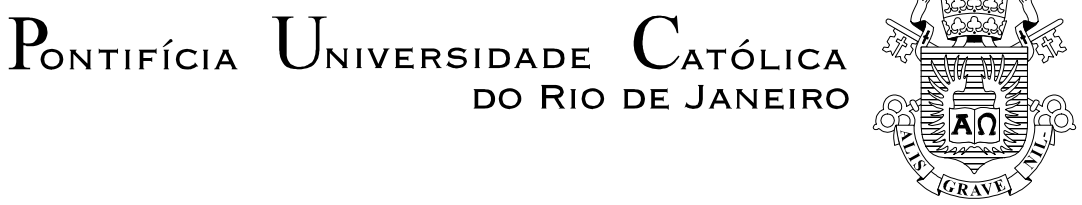

Fabíola Alice Alves Rocha dos Reis

\section{CRENÇAS SOBRE ENSINO E APRENDIZAGEM DE TRADUÇÃO: UMA REFLEXÃO BASEADA NOS ESTUDOS DA TRADUÇÃO E NA LINGUISTICA APLICADA}

Dissertação apresentada como requisito parcial para obtenção do grau de Mestre pelo programa de Pós-Graduação em Letras do Departamento de Letras do Centro de Teologia e Ciências Humanas da PUC-Rio. Aprovada pela Comissão Examinadora abaixo assinada.

Profa. Marcia do Amaral Peixoto Martins

Orientadora

Departamento de Letras - PUC-Rio

Profa. Sílvia Beatriz Alexandra Becher Costa

Departamento de Letras - PUC-Rio

Profa. Maria Alice Gonçalves Antunes

UERJ

Profa. Denise Berruezo Portinari Coordenadora Setorial do Centro de Teologia e Ciências Humanas - PUC-Rio

Rio de Janeiro, 9 de fevereiro de 2012. 
Todos os direitos reservados. É proibida a reprodução total ou parcial do trabalho sem autorização da universidade, da autora e do orientador.

\section{Fabíola Alice Alves Rocha dos Reis}

Cursou o Bacharelado em Letras (Português-Inglês) pela UFRJ. Licenciou-se em 2003. Ingressou na Formação de Tradutores Inglês-Português da PUC-Rio em 2005 e na Especialização em Tradução Inglês-Português da mesma instituição em 2007. Dedica-se ao magistério da língua inglesa desde 1998. Seus interesses incluem os Estudos da Tradução e a Linguística Aplicada ao Ensino de Línguas.

Ficha Catalográfica

Reis, Fabíola Alice Alves Rocha dos

Crenças sobre ensino e aprendizagem de tradução: uma reflexão baseada nos estudos da tradução e na linguística aplicada / Fabíola Alice Alves Rocha dos Reis ; orientadora: Marcia do Amaral Peixoto Martins. 2012.

109 f. : il. ; $30 \mathrm{~cm}$

Dissertação (mestrado)-Pontifícia Universidade Católica do Rio de Janeiro, Departamento de Letras, 2012.

Inclui bibliografia

1. Letras - Teses. 2. Tradução. 3. Ensino. 4. Aprendizagem. 5. Crenças. 6. Pedagogia da tradução. 7. Formação do tradutor. I. Martins, Marcia do Amaral Peixoto. II. Pontifícia Universidade Católica do Rio de Janeiro. Departamento de Letras. III. Título. 


\section{Agradecimentos}

\section{A Deus}

Ao meu filho João Pedro, que foi gestado ao mesmo tempo que esta dissertação, por me fazer querer ser uma pessoa melhor

Ao meu marido Ricardo, pela nossa história e pelo apoio contínuo

Aos meus pais Luiz Antônio e Miriam, pelo dom da vida

Aos meus avós Maria Luiza, Ricardo, Maria e José (in memoriam), por serem meu porto seguro, hoje e sempre

À professora Márcia do Amaral Peixoto Martins, pela orientação clara e por todo apoio

Às professoras da banca examinadora, Silvia Becher, Maria Alice Antunes e Maria Paula Frota

Às professoras Maria Paula Frota e Inés Kayón de Miller, por toda ajuda oferecida

A todos os professores que tive

Aos informantes que aceitaram participar desta pesquisa

A Francisca Ferreira de Oliveira (Chiquinha) e a todos os funcionários da Secretaria de Pós-Graduação do Departamento de Letras

À PUC-Rio, pela concessão da bolsa de isenção de mensalidade 


\section{Resumo}

Reis, Fabíola Alice Alves Rocha dos; Martins, Marcia do Amaral Peixoto. Crenças sobre ensino e aprendizagem de tradução: uma reflexão baseada nos Estudos da Tradução e na Linguística Aplicada. Rio de Janeiro, 2012. 109 p. Dissertação de Mestrado - Departamento de Letras, Pontifícia Universidade Católica do Rio de Janeiro.

A crescente expansão da atividade tradutória no mundo, bem como o surgimento de novas modalidades de tradução, tem gerado um aumento na oferta de cursos de formação de tradutores, nos mais diversos níveis. No entanto, apenas nos últimos anos os estudos voltados especificamente para a pedagogia da tradução se intensificaram. Esta pesquisa qualitativa investiga as crenças sobre ensino e aprendizagem de Tradução de dois pequenos grupos de informantes: alunos iniciantes do Bacharelado em Letras - habilitação em Tradução da Pontifícia Universidade Católica do Rio de Janeiro (PUC-Rio), todos sem experiência anterior na atividade, e tradutores novatos, egressos ou graduandos desse mesmo curso, que já começaram a atuar profissionalmente. A análise das entrevistas realizadas com os informantes se apoia na literatura acadêmica dos Estudos da Tradução, além das pesquisas sobre a sala de aula de língua estrangeira advindas da Linguística Aplicada. As conclusões indicam crenças que mostram a importância da teoria aplicada à prática, do professor, e da aproximação entre a prática pedagógica empregada no curso e a realidade do mercado de trabalho.

\section{Palavras-chave}

tradução; ensino; aprendizagem; crenças; pedagogia da tradução; formação do tradutor 


\section{Abstract}

Reis, Fabíola Alice Alves Rocha dos; Martins, Marcia do Amaral Peixoto (Advisor). Beliefs about translation teaching and learning: a reflection based upon Translation Studies and Applied Linguistics. Rio de Janeiro, 2012. 109 p. Dissertação de Mestrado - Departamento de Letras, Pontifícia Universidade Católica do Rio de Janeiro.

The amount of existing translator-training courses has increased dramatically over the years due to the rising number of translation commissions and new translation modalities. However, not until the last few years have the number of studies specifically dedicated to Translation pedagogy risen as well. This qualitative research investigates the beliefs about translation teaching and learning from two small groups of interviewees: beginner students of Translation, with no professional experience in the area, and recently graduated students, who have already started translating professionally. All of them have taken the Translation graduation programme at the Pontifícia Universidade Católica (PUC-Rio). The analysis is based on the academic literature from the Translation Studies field, in addition to the research about the foreign language classroom coming from the Applied Linguistics field. Conclusions highlight beliefs that show how important translation theory apllied to practice is, how important the professor is and how important it is to have a pedagogical practice that reflects the job market.

\section{Keywords}

translation; teaching; learning; beliefs; Translation pedagogy; translation training 


\section{Sumário}

$\begin{array}{ll}\text { 1. Introdução } & 10\end{array}$

2. Fundamentação teórica e metodologia de pesquisa 14

2.1. Diferentes abordagens aplicadas ao ensino de tradução 14

2.1.1. A abordagem transmissionista 17

2.1.2. A abordagem cognitivista 20

2.1.3 A abordagem pós-estruturalista 21

2.1.4 A abordagem socioconstrutivista 23

2.2 A questão das crenças à luz dos Estudos da Tradução 26

2.3 A questão das crenças à luz da LAELE 28

2.4 Metodologia de pesquisa 33

2.4.1 Apresentação dos informantes 39

3. Análise dos dados 42

3.1 Primeira questão mencionada pelo entrevistado 43

3.2 Práticas pedagógicas $\quad 49$

3.30 papel do professor $\quad 57$

3.4Considerações finais $\quad 61$

4. Conclusão 64

5. Referências bibliográficas 69

6. Anexos 72

6.1. Roteiro semiestruturado de perguntas $\quad 72$

6.2. Transcrição das entrevistas 73 


\section{Siglas}

$\mathrm{AI}$ - aprendiz iniciante

CNPq - Conselho Nacional de Desenvolvimento Científico e Tecnológico

Enade - Exame Nacional de Desempenho de Estudantes

LAELE - Linguística Aplicada ao Ensino de Língua Estrangeira

PUC - Pontifícia Universidade Católica

TAPs - Think-Aloud Protocols ou protocolos verbais

$\mathrm{TN}$ - tradutor novato

UFRGS - Universidade Federal do Rio Grande do Sul

UFRJ - Universidade Federal do Rio de Janeiro 
